\title{
Image detection and verification of visual navigation route during cotton field management period
}

\author{
Jingbin $\mathrm{Li}^{1,2}$, Rongguang $\mathrm{Zhu}^{1,2^{*}}$, Bingqi Chen ${ }^{3}$ \\ (1. College of Mechanical and Electrical Engineering, Shihezi University, Shihezi 832003, China; \\ 2. Key Laboratory for Agricultural Machinery of Xinjiang BINGTUAN, Shihezi University, Shihezi 832003, China; \\ 3. College of Engineering, China agricultural University, Beijing 100083, China)
}

\begin{abstract}
In order to meet the actual operation demand of visual navigation during cotton field management period, image detection algorithm of visual navigation route during this period was investigated in this research. Firstly, for the operation images under natural environment, the approach of color component difference, which is applicable for cotton field management, was adopted to extract the target characteristics of different regions inside and outside cotton field. Secondly, the median filtering method was employed to eliminate noise in the images and realize smoothing process of the images. Then, according to the regional vertical cumulative distribution graph of the images, the boundary characteristic of the cotton seedling region was obtained and the central position of the cotton seedling row was determined. Finally, the detection of the candidate points cluster was realized, and the navigation route was extracted by Hough transformation passing the known point. The testing results showed that the algorithms could rapidly and accurately detect the navigation route during cotton field management period. And the average processing time periods for each frame image at the emergence, strong seedling, budding and blooming stages were $41.43 \mathrm{~ms}, 67.83 \mathrm{~ms}, 68.80 \mathrm{~ms}$ and $74.05 \mathrm{~ms}$, respectively. The detection has the advantage of high accuracy, strong robustness and fast speed, and is simultaneously less vulnerable to interference from external environment, which satisfies the practical operation requirements of cotton field management machinery.
\end{abstract}

Keywords: visual navigation, route detection, Hough transformation passing the known point, cotton field management period DOI: $10.25165 /$ j.ijabe.20181106.3976

Citation: Li J B, Zhu R G, Chen B Q. Image detection and verification of visual navigation route during cotton field management period. Int J Agric \& Biol Eng, 2018; 11(6): 159-165.

\section{Introduction}

Visual navigation is one common way of automatic navigation in agriculture, and has become a research hotspot in the field of automatic navigation. Currently, many research achievements have been acquired in detection methods of navigation route in China and abroad, and these achievements mainly focus on detection of crops, such as rice, wheat, and corn, involving operation processes of cultivating, crop protection, sowing, and harvesting. The detection approaches mainly include: the method of extracting crop row based on the Hough transformation and obtaining parameters of crop row and navigation ${ }^{[1-3]}$; the procedure of detecting crops and obtaining their central line ${ }^{[4,5]}$; the technique of separating crop and background by filtering characteristic values and obtaining crop central line by robust linear fitting ${ }^{[6]}$; the method of extracting central line of rice seedling row based on color model and nearest neighbor clustering ${ }^{[7]}$; the approach of implementing rotary projection algorithm to image ROIs by angle enumeration ${ }^{[8]}$; the method of detecting row center of crop at early stage based on least squares method ${ }^{[9]}$; the technique of using the classification algorithm based on horizontal line scanning to solve

\section{Received date: 2017-11-16 Accepted date: 2018-04-16}

Biographies: Jingbin $\mathbf{L i}, \mathrm{PhD}$, Professor, research interests: image processing and machine vision, Email: 1jb8095@163.com; Bingqi Chen, PhD, Professor, research interests: image processing and machine vision, Email: fbcbq@ 163.com.

*Corresponding author: Rongguang Zhu, $\mathrm{PhD}$, Associate Professor, research interests: application of rapid optical analysis techniques. College of Mechanical and Electrical Engineering, Shihezi University, Shihezi 832003, China. Tel: +86-18999537120, Email: rgzh_jd@163.com. the multiple target detection problem of farmland images; and the method of utilizing improved Hough transformation to rapidly detect multiple positioning lines ${ }^{[10]}$. Moreover, Chen et al. ${ }^{[11-17]}$ has thoroughly studied the visual system of the rice transplanting robot and the route detection of rice field management and farming robot. $\mathrm{Li}$ et al. ${ }^{[18,19]}$ studied the machine vision detection of navigation route of the cotton planter and harvester. Haw and Ismail ${ }^{[20]}$ studied the colour vision and image processing technique to determine paddy maturity. However, at present, few detection approaches for navigation route during cotton field management period in China have been reported.

This research aimed at analyzing and processing the color images shot under natural environment during cotton field management period, and then extracting the navigation route for operation of cotton field management machinery.

\section{Experimental methods}

\subsection{Experimental equipment and image acquisition}

The operation diagram for collecting images during cotton field management period is shown in Figure 1. The tractor 754 (John Deere China, Beijing, China) was adopted and Camera T60 (Aigo, Beijing, China) was used for image collection. The camera was mounted at the middle position in front of the tractor and its installation height was adjustable according to the cotton plant height. The angle between the camera axle and the horizontal plane was $20^{\circ}$ downwards. The output color image had a resolution of $640 \times 480$ pixels. VS2010 (Microsoft, USA) was utilized for developing algorithm.

The image data in the experiment was collected between May and September, 2013, in Regiment 147, Shihezi, Xinjiang 
Production and Construction Corps, China.

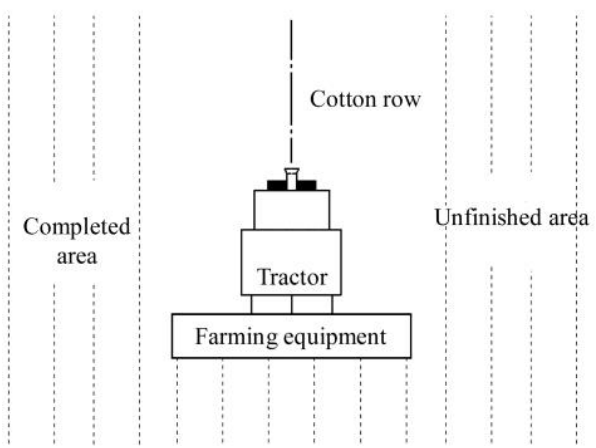

Figure 1 Operation diagram of cotton field management machinery

2.2 Detection of candidate points cluster of first frame image and navigation route

1) The image processing window is shown in Figure 2, and it was the region rounded up by four points of $(s x, 0),(e x, 0),(s x$, ysize) and (ex, ysize $)$ in the plane coordinate system. At the stages of emergence, strong seedling and budding, the width of $s x$ and $e x$ was set as $3 / 8$ and $5 / 8$ of that of xsize, respectively; and at the blooming stage, the width of $s x$ and $e x$ was set as $1 / 4$ and $3 / 4$ of that of xsize, respectively.

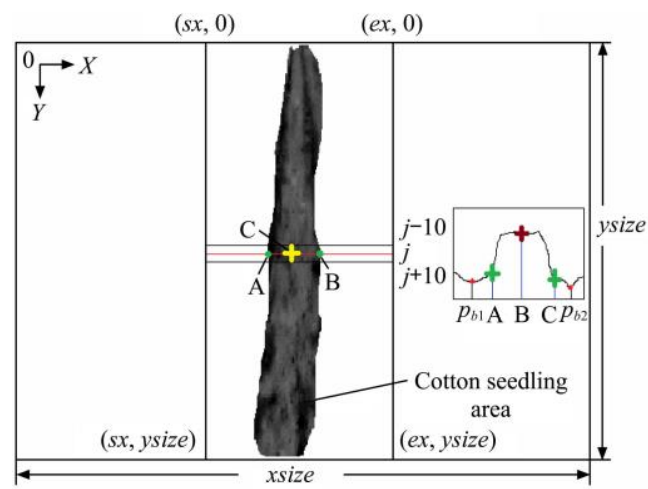

Note: $p_{b 1}$ and $p_{b 2}$ represent the lowest trough points at left side and right side of array $Q ; \mathrm{A}$ and B represent the critical points along the upper edges at left and right sides; $\mathrm{C}$ indicates the candidate point

Figure 2 Schematic diagram of image processing window and Candidate points detection

2) The target image in the processing window was scanned, and the $2 \mathrm{G}-\mathrm{R}-\mathrm{B}$ value of each pixel was brought into $2 \mathrm{G}-\mathrm{R}-\mathrm{B}$ model formula (1) to calculate the brightness value $E$.

$$
E=2 \times G-R-B
$$

3) The target window (the floating window) was set and its pixel values in vertical direction were accumulated. In the target window, the floating window was set with height of $[j-10, j+10]$, where $10 \leq j \leq y$ size -10 , which is the middle rectangle box as shown in the middle of Figure 2. Arrays of $Q[d]$ and $Q_{1}[d]$ were created, and their size was set as $d=e x-s x+1$. In the floating window, all pixels were scanned row by row, and their brightness values were vertically accumulated to $Q_{1}$.

4) As shown in Figure 2, array $Q_{1}$ was smoothed by moving average method, and the data after smoothing was recorded in array $Q$.

5) Equations (2) and (3) were employed to solve the average value $\overline{E_{1}}$ and standard deviation $D$ of the array $Q$ of brightness values.

$$
\bar{E}_{1}=\frac{1}{d} \sum_{i=0}^{d} L[i]
$$

$$
D=\sqrt{\frac{1}{d-1} \sum_{i=0}^{d}\left(L[i]-\bar{E}_{1}\right)^{2}}
$$

6) The lowest trough points $p_{b 1}$ and $p_{b 2}$ at both sides of array $Q$ were searched for by taking $d / 2$ as the center. The search for lowest trough point at left side was taken as the example. Initial value was set as $b_{\min }=\bar{E}_{1}$. Array $Q$ was scanned from $i=0$ $(0 \leq i \leq(e x-s x+1) / 2))$. When $Q[i] \leq b_{\min }$, it was recorded that $b_{\min }=Q[i]$ and $p_{1}=i+s x$. After finishing the scan, $p_{b 1}=p_{1}$ was the position of the lowest trough point at left side of array $Q$. Similarly, array $Q$ was scanned from $i=(e x-s x+1)((e x-s x+1) / 2 \leq i \leq$ $e x-s x)$ to search for the lowest trough point $p_{b 2}$ at right side of $Q$. As shown in Figure 2, $p_{b 1}$ and $p_{b 2}$ are the lowest trough points.

7) Critical points along the upper edge of peak were searched for in the direction from lowest trough points to cotton seedling region, and these points were edge pixels at the boundary. The search for critical point along the upper edge of peak in the direction from the lowest trough point at left side to cotton seedling region was taken as the example. Threshold was set as $T=\varepsilon \times D(\varepsilon$ had different values at different stages), and array $Q$ was scanned from $p=p_{b 1} \quad\left(p_{b} \leq p \leq(e x-s x+1) / 2\right.$. When $\left|Q[p]-b_{\min }\right|>T$, it was recorded that $p_{2}=p+s x$. When $\left|Q[p]-b_{\min }\right|>T$, the scanning was stopped. Thus, the critical point along the upper edge of peak at left side was $p_{l}=p_{2}$, which is point $\mathrm{A}$ as shown in Figure 2.

8) According to critical points of $p_{l}$ and $p_{r}$ at both left and right sides of cotton seedling region, candidate point $p_{p}$ was solved by formula (4), which is point $\mathrm{C}$ as shown in Figure 2.

$$
p_{p}=\frac{p_{l}+p_{r}}{2}
$$

9) By repeatedly performing step (4) to step (8), the cluster of candidate points at the central line of cotton seedling row could be obtained. As shown in Figure 3, the number was set as $\mathrm{n}$.

10) Known point $(X, Y)$ was determined. The coordinates of candidate points at the boundary were set as $\left(x_{u}, y_{u}\right)$, where $0 \leq u \leq n$. Formula (5) was then used to solve $(X, Y)$, which is at the position of "+" as shown in Figure 3.

$$
\left\{\begin{array}{l}
X=\frac{1}{n} \sum_{u=1}^{n} x_{u} \\
Y=\frac{1}{2} \text { ysize }
\end{array}\right.
$$

11) The candidate point cluster acquired was taken as the target. By using Hough transformation passing the known point to perform straight line fitting, the navigation path was fitted and obtained. Meanwhile, the data of all points on the navigation path was recorded into array $V[j]$.

\subsection{Detection of candidate points cluster of non-first frame image and navigation path}

After the first frame image, image of each frame was associated with the previous adjacent frame. Firstly, target image region was processed by median filtering. Then, the processing region was re-determined according to the known point acquired from the image of previous frame before it. Finally, detection algorithm of candidate point cluster was performed to lock the candidate point cluster for the current frame image.

1) Starting from the second frame image, the processing window was set as $s x=X-x$ size $/ 8, e x=X+x$ size $/ 8$.

2) Steps of (2) to (6) in part 1.2 of the detection algorithm of first frame image, were repeatedly performed.

3) Critical points along the upper edge of peak were searched for in the direction from lowest trough point to cotton seedling 
region. The data of array V[ysize] of previous frame image by straight line detection was taken as that of the central point. Then from the point, $\mathrm{m}$ pixels were expanded towards left and right, respectively. If $\left|p_{2}-V[j]\right| \leq m, p_{p}=p_{2}$, or $p_{p}=V[j]$.

where, $m=\tan \alpha \times y$ size, and $\alpha=3^{\circ}$, which was the maximum deviation angle allowed for operation of field management machinery. A region of $2 \mathrm{~m}$ pixels is shown as in Figure 3.

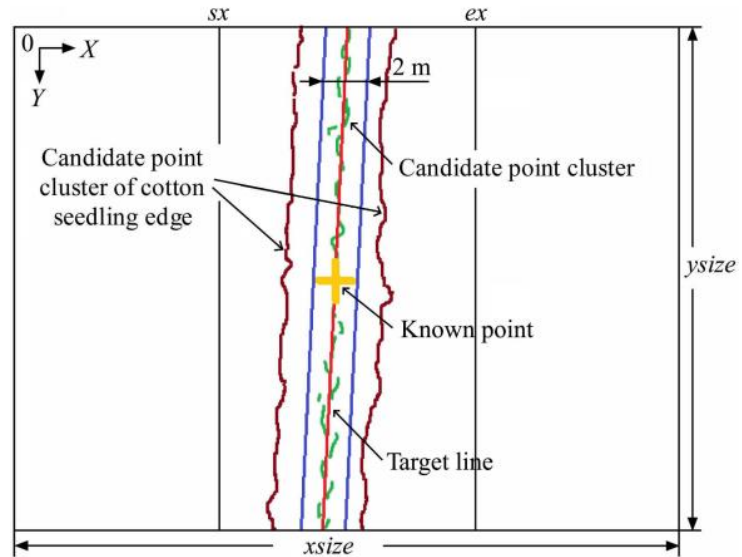

Figure 3 Detection diagram of candidate points cluster, the known point and the navigation line
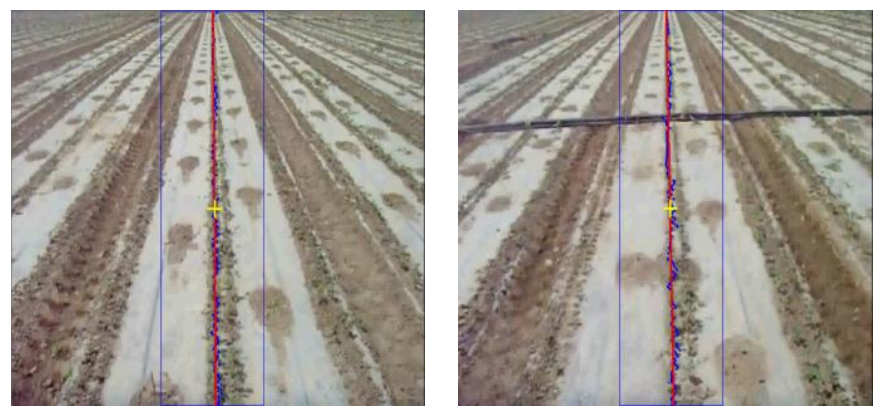

a. Flat land surface, and clear navigation b. Drip irrigation belt branches distributed target in the field

4) Steps of (8) to (11) in part 1.2 of the detection algorithm of first frame image, were repeatedly performed to realize the detection of navigation path.

\section{Experimental results and analysis}

\subsection{Detection of navigation route during field management period}

\subsubsection{Emergence stage}

For the visual navigation route detection at the emergence stage, detection algorithm of candidate point cluster during sowing period was adopted ${ }^{[18,19]}$. For visual navigation route detection at the strong seedling stage, budding stage, and blooming stage, detection algorithm of candidate point cluster based on the characteristic of central line of cotton seedling row was adopted. Hough transformation passing the known point was then utilized to fit navigation route. The original images and detection results at the emergence stage are shown in Figure 4.

All images in Figure 4 were collected between 10:00 am and 2:00 pm. Figure 4a was of flat land surface and had clear navigation object characteristic; Figure $4 \mathrm{~b}$ was of flat land surface and had clear navigation target characteristic, but had water supply branches of drip irrigation belt distributed in the middle; Figure $4 \mathrm{c}$ contained weeds in the field; Figure $4 \mathrm{~d}$ had the break characteristic of soil-covering rows for cotton seedling due to weather reason.

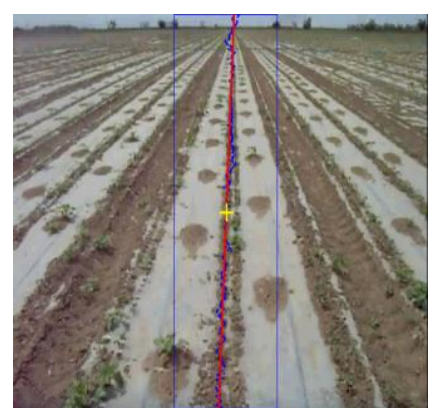

c. Weeds in the field

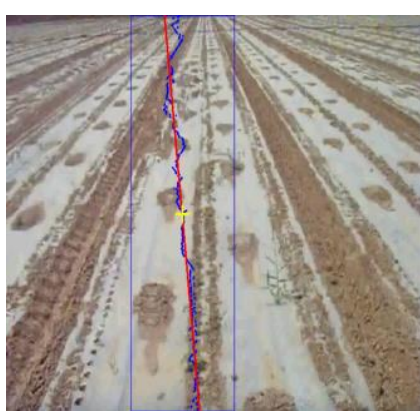

d. Soil-covering characteristic broke

Figure 4 Original images and detection results at the emergence stage

Figure 5 shows the result comparison of each image in Figure 4 before and after smoothing with Daubechies wavelet. From Figures $5 \mathrm{a}-5 \mathrm{c}$, it can be seen that the noise in the images was reduced obviously after Daubechies wavelet smoothing. Especially for the region near the soil-covering seed hole, which was chosen as the navigation target characteristic, its color distribution had a shape of double-bottom before smoothing, and then became the shape of single-trough after smoothing. Therefore, the candidate point cluster could be accurately found out. It can be seen from Figure $5 \mathrm{~d}$ that trough characteristic encountered serious deviation at the disappearing region of soil-covering characteristic. Therefore, the candidate point cluster detected seriously deviated from practical proceeding route, and thus the navigation straight line detected also severely deviated from the practical route.

From the detection results in Figures $4 \mathrm{a}-4 \mathrm{c}$, it can be seen that, the candidate point clusters of all images, and navigation straight lines detected according to Hough transformation passing the known point totally agreed with the navigation target characteristic. Moreover, from Figures $4 \mathrm{~b}$ and $4 \mathrm{c}$, it can be seen that this detection algorithm would not be affected by isolated noise of drip irrigation belt branches and weeds, and had strong robustness. And from Figure $4 \mathrm{~d}$, it can be seen that the navigation target characteristic broke, so the navigation straight line deviated.

\subsubsection{Strong seedling stage}

The original images of chemical control operation at the strong seedling stage of cotton and their detection results for navigation route are shown in Figure 6. In Figure 6a, cotton rows were in order and the film shadow existed at left side of cotton row; in Figure $6 \mathrm{~b}$, the phenomenon of seedling shortage or row breaks existed in cotton field.

Figures $7 \mathrm{a}$ and $7 \mathrm{c}$ are the grayscale image based on $2 \mathrm{G}-\mathrm{R}-\mathrm{B}$ color model and its vertical cumulative distribution graph of Figures $6 \mathrm{a}$ and $6 \mathrm{~b}$, respectively. Figures $7 \mathrm{~b}$ and $7 \mathrm{~d}$ are the filtered image based on moving average method and its vertical cumulative distribution graph of Figures $7 \mathrm{a}$ and $7 \mathrm{c}$, respectively. From Figure $7 \mathrm{a}$, it can be seen that, after being processed by $2 \mathrm{G}-\mathrm{R}-\mathrm{B}$ color model, the green characteristic of the image was intensified and the film and shadow characteristics were effectively suppressed. From Figure 7c, it can be seen that, at the breaking part of cotton row, the cumulative grayscale value decreased sharply, while the soil-covering region still had certain degree of navigation characteristic. And the cumulative distribution graph met the detection algorithm for central line of cotton row. Therefore, it could still detect the candidate point cluster to some extent. From Figures $7 \mathrm{~b}$ and $7 \mathrm{~d}$, it can be seen that, after filtering, sharp noise was eliminated and that laid the foundation for extraction of later boundary pixels. 
From the point cluster distribution at left and right boundaries of cotton seedling row in Figure 6, it can be seen that the point clusters detected satisfied the left and right boundaries. The candidate point cluster detected in the middle met the central line characteristic of cotton seedling row. From Figure $6 \mathrm{~b}$, it can be
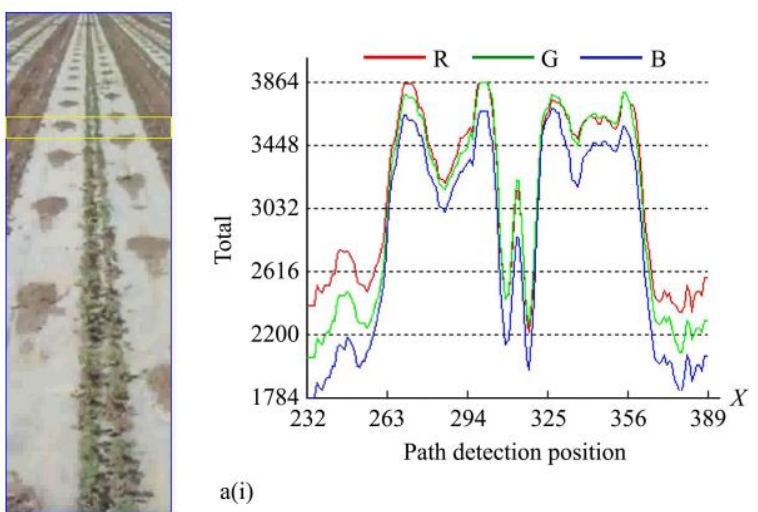

a(i)
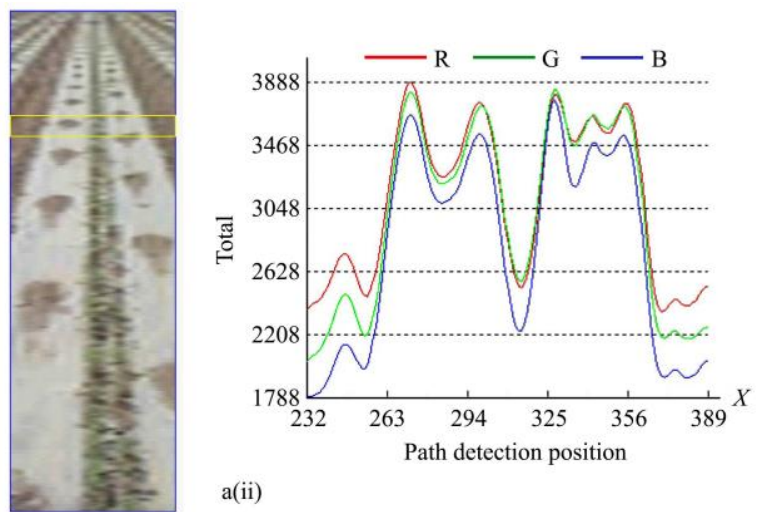

a. Comparison of color distribution at image processing area in Figure 4a before a(i) and after a(ii) Daubechies wavelet
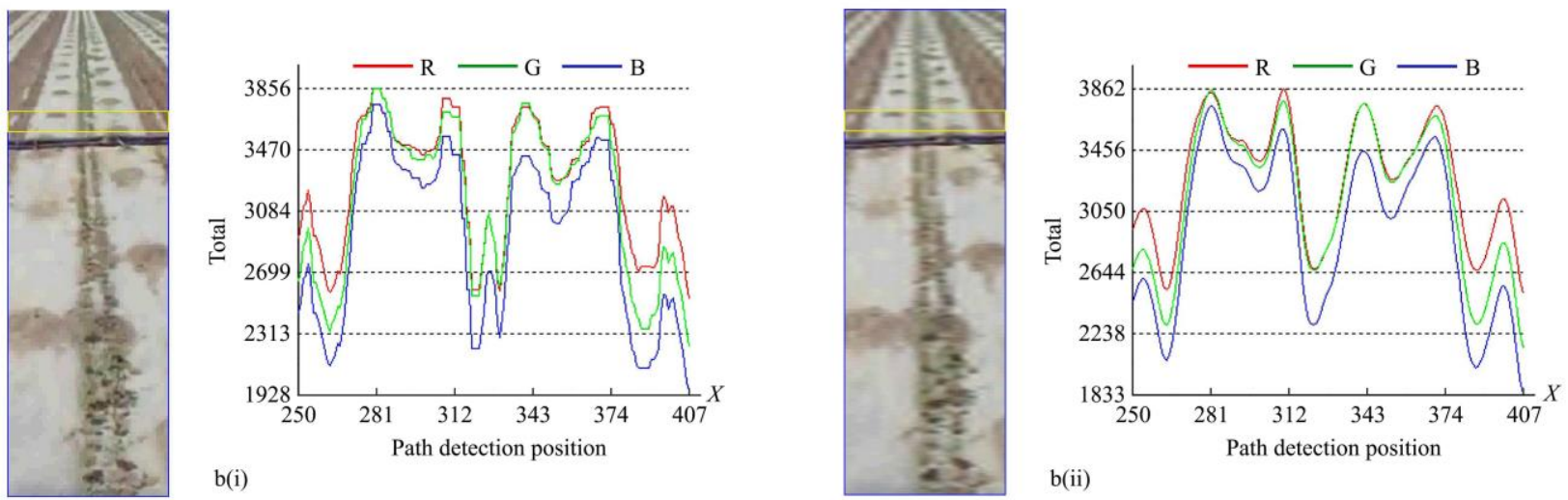

b. Comparison of color distribution at image processing area in Figure $4 \mathrm{~b}$ before $b(\mathrm{i})$ and after b(ii) Daubechies wavelet
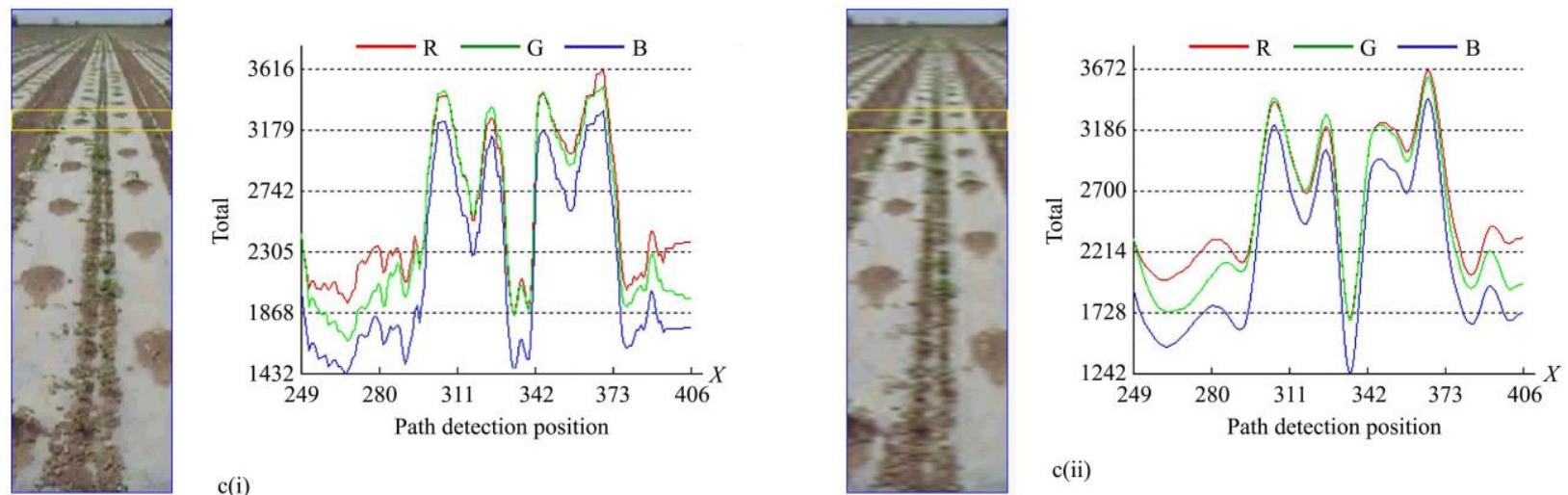

c(i)

c(ii)

c. Comparison of color distribution at image processing area in Figure $4 \mathrm{c}$ before c(i) and after c(ii) Daubechies wavelet
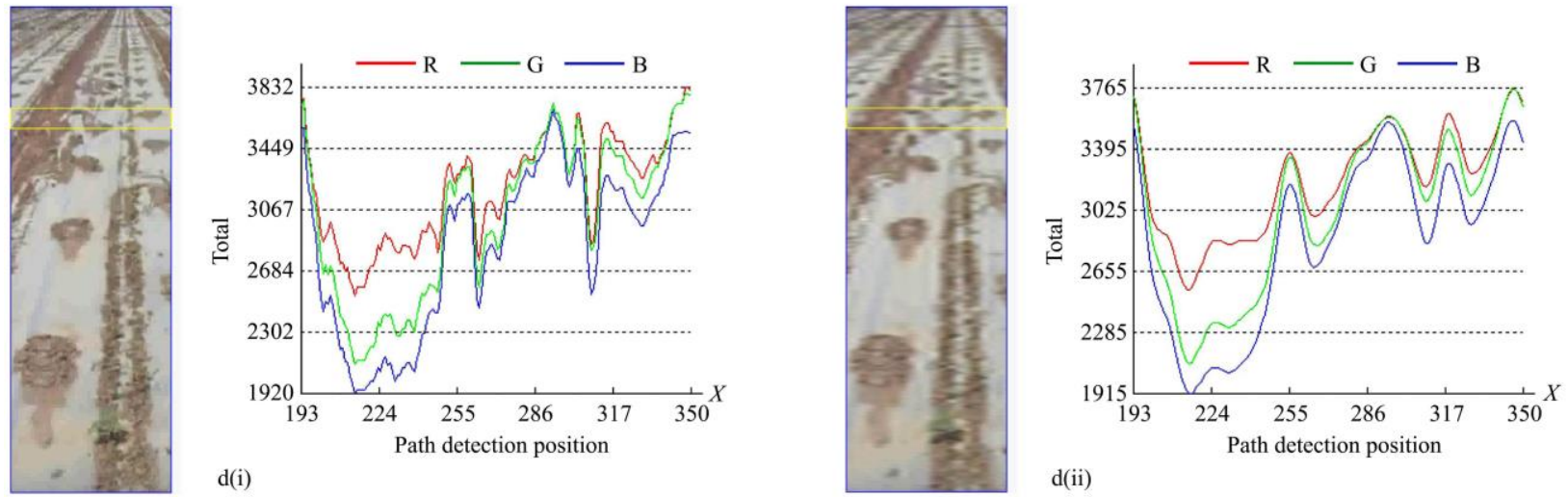

d. Comparison of color distribution at image processing area in Figure $4 \mathrm{~d}$ before d(i) and after d(ii) Daubechies wavelet

Figure 5 Comparison of color distribution at image processing areas in Figure 4 before and after Daubechies wavelet 


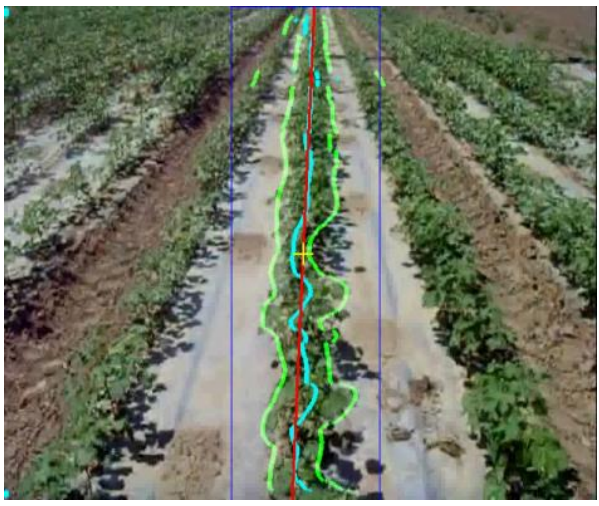

a. Cotton rows were in order and the film shadow existed

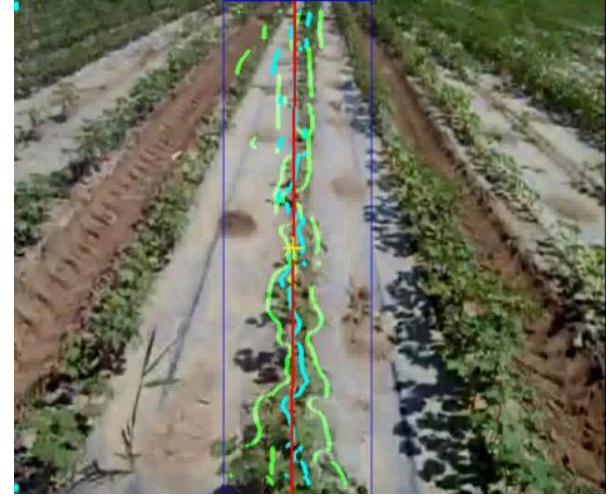

b. Cotton row was broke

Figure 6 Original images and detection results at strong seedling stage
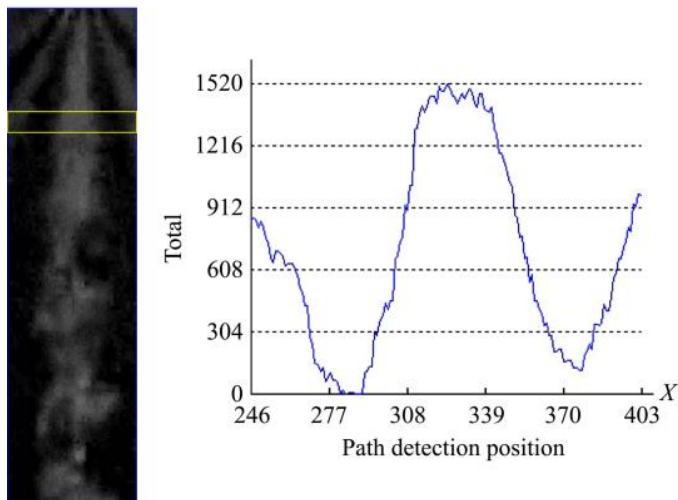

a. Grayscale image of Figure 6a
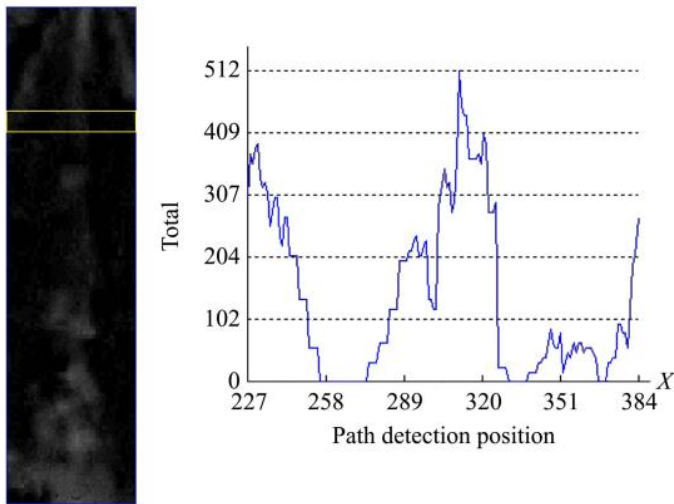

c. Grayscale image of Figure $6 \mathrm{~b}$
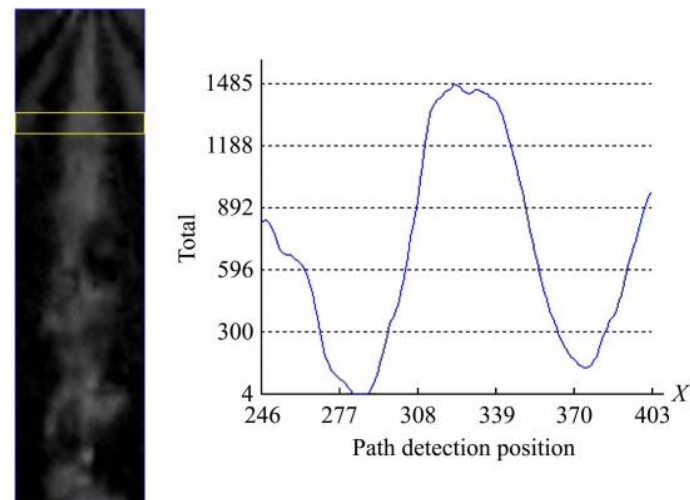

b. Filtered image of Figure 7a
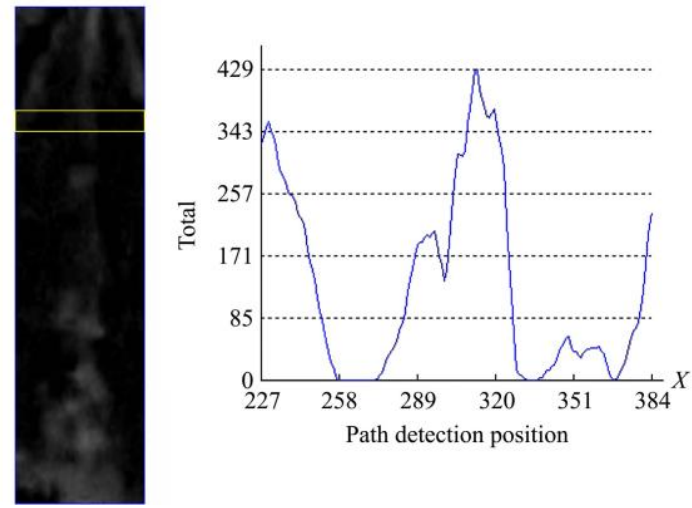

d. Filtered image of Figure 7c

Figure 7 Results comparison of grayscale and filtered images of Figure 6

Moreover, from the detection results of visual navigation straight line in Figure 6, it can be seen that navigation straight line detected by this algorithm accorded with the central line characteristic of cotton seedling row. And the algorithm carried out the detection based on the relevance of two adjacent frames, so the robustness and availability of the algorithm could be effectively improved.

\subsubsection{Budding and blooming stage}

The original images of operation and their detection results at the budding stage and blooming stage are shown in Figure 8. Figure $8 \mathrm{a}$ is the image collected at the budding stage. The row lines of cotton seedling were straight, film and land surface was exposed, and some cotton shadow existed in intervals between cotton seedling lines. Figure $8 \mathrm{~b}$ is another image collected at the budding stage. Lots of straws and cotton shadow scattered in intervals between seedling lines, and film and land surface were exposed. Figure $8 \mathrm{c}$ is the image collected at the blooming stage. The row lines of cotton seedling were straight and a great deal of shadow existed in intervals at the left side. Figure $8 \mathrm{~d}$ is another image collected at the blooming stage. The cotton seedlings at the upper area of the image were stronger than those at the lower area, which were replanted as supplementation.

From the detection results of candidate point cluster in Figure 8 , it can be seen that the algorithm developed could correctly extract the boundary point characteristic of the cotton row during this period, thus to find out the candidate point cluster for visual navigation. The candidate point cluster detected met the central line characteristic of the cotton row. And the detection algorithm based on the relevance of two adjacent frames could guarantee that the candidate point clusters be intensively and continuously distributed.

From the detection results of navigation straight line in Figure 8 , it can be seen that the candidate points cluster found on the basis of central line characteristic of cotton seedling row and navigation straight line detected according to Hough transformation passing the known point accorded with the central line characteristic of 
cotton seedling rows.

\subsection{Experimental verification}

In order to verify the real-time performance and accuracy of detection algorithm for visual navigation route during cotton field

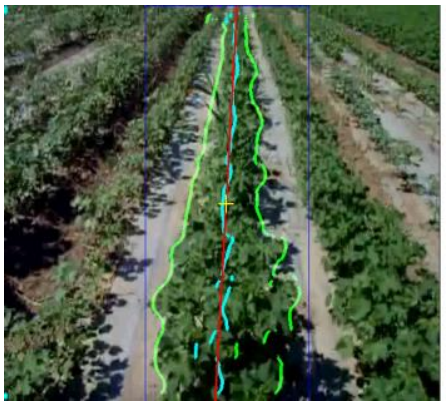

a. Budding stage

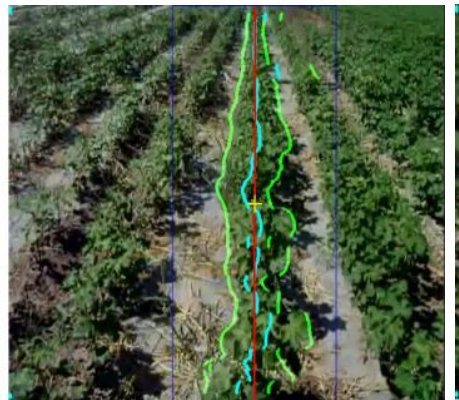

b. Budding stage, with straws

management period, real-time operation videos collected at each stage of field management period were processed, and frames with detection error and their processing speeds were statistically analyzed. The results are shown in Table 1.

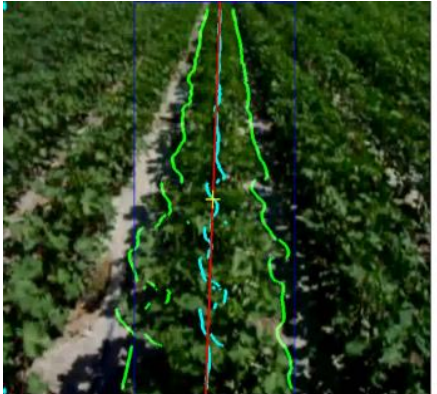

c. Blooming stage

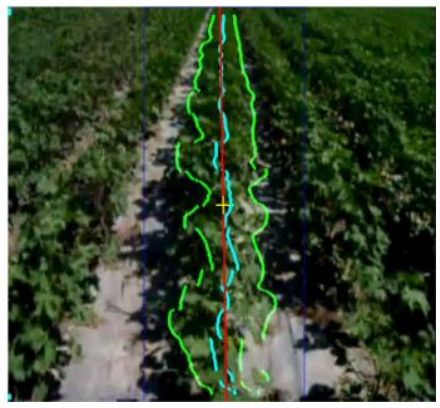

d. Blooming stage, with small cotton seedlings

Figure 8 Original images and detection results at the budding and blooming stages

Table 1 Experimental results of detection algorithm for visual navigation route during cotton field management period

\begin{tabular}{|c|c|c|c|c|c|c|}
\hline $\begin{array}{l}\text { Field management } \\
\text { links }\end{array}$ & $\begin{array}{c}\text { Video } \\
\text { Sequence number }\end{array}$ & $\begin{array}{l}\text { Video } \\
\text { frames }\end{array}$ & $\begin{array}{l}\text { The starting frame of } \\
\text { detection error (frame) }\end{array}$ & $\begin{array}{l}\text { Average processing } \\
\text { speed } /(\mathrm{ms} / \text { frame })\end{array}$ & Accuracy $/ \%$ & Note \\
\hline \multirow{8}{*}{ Emergence stage } & 1 & 2401 & l & 42.27 & 100 & \multirow{8}{*}{$\begin{array}{l}\text { For videos } 1-6 \text {, soil-covering characteristics } \\
\text { were obvious; for videos } 7-8 \text {, soil-covering } \\
\text { characteristics disappeared and the } \\
\text { detection was stopped. }\end{array}$} \\
\hline & 2 & 2000 & l & 40.46 & 100 & \\
\hline & 3 & 3948 & l & 41.4 & 100 & \\
\hline & 4 & 4601 & I & 41.02 & 100 & \\
\hline & 5 & 3346 & l & 42.03 & 100 & \\
\hline & 6 & 2845 & l & 41.56 & 100 & \\
\hline & 7 & 1844 & 1481 & 41.73 & I & \\
\hline & 8 & 2860 & 2561 & 41.01 & l & \\
\hline \multirow{6}{*}{ Strong seedling stage } & 1 & 601 & l & 67.9 & 100 & \multirow{6}{*}{$\begin{array}{l}\text { For videos } 1-5 \text {, cotton seedling rows were } \\
\text { consecutive; for video } 6 \text {, cotton seedling } \\
\text { row was inconsecutive and the detection } \\
\text { accuracy was less than } 100 \% \text {. }\end{array}$} \\
\hline & 2 & 1051 & l & 67.1 & 100 & \\
\hline & 3 & 1801 & I & 68.42 & 100 & \\
\hline & 4 & 2696 & l & 67.56 & 100 & \\
\hline & 5 & 3824 & l & 67.82 & 100 & \\
\hline & 6 & 3219 & 2815 & 68.23 & l & \\
\hline \multirow{6}{*}{ Budding stage } & 1 & 1502 & l & 68.86 & 100 & \multirow{6}{*}{$\begin{array}{l}\text { For all the videos, cotton seedling rows } \\
\text { were consecutive. }\end{array}$} \\
\hline & 2 & 1301 & I & 69.94 & 100 & \\
\hline & 3 & 1852 & I & 68.35 & 100 & \\
\hline & 4 & 2446 & I & 68.63 & 100 & \\
\hline & 5 & 3128 & l & 69.32 & 100 & \\
\hline & 6 & 2638 & l & 67.75 & 100 & \\
\hline \multirow{6}{*}{ Blooming stage } & 1 & 6452 & l & 77.63 & 100 & \multirow{6}{*}{$\begin{array}{l}\text { For all the videos, cotton seedling rows } \\
\text { were consecutive. }\end{array}$} \\
\hline & 2 & 4501 & l & 73.93 & 100 & \\
\hline & 3 & 3000 & I & 72.31 & 100 & \\
\hline & 4 & 2301 & l & 75 & 100 & \\
\hline & 5 & 2001 & I & 72.81 & 100 & \\
\hline & 6 & 1701 & l & 72.62 & 100 & \\
\hline
\end{tabular}

From Table 1 it can be seen that, at the emergence stage, navigation route of film mulch planter was effectively detected by using detection algorithm for visual navigation route, and the average detection time was $41.43 \mathrm{~ms}$ for each frame image. Based on central line characteristic of cotton seedling row, candidate points were detected by using candidate point cluster detection algorithm. Then visual navigation routes at the strong seedling stage, budding stage and blooming stage, were effectively and accurately detected based on Hough transformation passing the known point, respectively. The average detection time for each frame image at strong seedling stage, budding stage and blooming stage was $67.88 \mathrm{~ms}, 68.73 \mathrm{~ms}$, and $74.78 \mathrm{~ms}$, respectively. The experimental results showed that the detection algorithm developed for visual navigation route during field management period was of high accuracy and fast detection speed, and could meet real-time operation requirements at different stages during cotton field management period.

From the list of starting frame of detection errors in Table 1, it can be seen that, due to disappearance of soil-covering characteristic of seed holes as well as break of cotton rows, the 
detection of navigation line would be affected and error occurred at the emergence and strong seedling stages.

In actual detection process, the detection of field end was simultaneously implemented with the detection of navigation route. When the aforementioned characteristic was encountered, field end detection was carried out to remind drivers of changing driving mode so that proceeding route of operation machine could be guaranteed.

\section{Conclusions}

1) The procedure for extraction of navigation route during cotton field management period was developed. Firstly, the grayscale images were obtained, and smoothing process was made with the median filtering method. Secondly, boundary characteristic of the cotton seedling area was found according to the regional vertical cumulative distribution graph. Then, the central position of cotton seedling row was determined and the candidate point cluster detection was realized. Finally, navigation straight line was fitted through the Hough transformation passing the known point.

2) Testing results showed that detection time for each frame image was less than $75 \mathrm{~ms}$ during cotton field management period, which can meet the real-time operation requirements of agricultural plant protection machinery.

3) The navigation route extracted by detection algorithm for navigation straight line can accurately match the visual navigation target characteristic at each stage. Moreover, the detection has the advantage of high accuracy, strong robustness and fast speed, and is simultaneously less vulnerable to interference from external environment, which can meet the actual operation requirements of agricultural machinery.

\section{Acknowledgements}

This work has been financially supported by the National Natural Science Foundation of China (Grant No.31071329) and the Team Construction of Young and Middle-aged Talents in Science and Technology Innovation of Xinjiang Corps ( Grant No.2016BC001)

\section{[References]}

[1] Reid J F, Searcy S W. Detecting crop rows using the Hough transform. American Society of Agricultural Engineers. Microfiche collection (USA), 1986.

[2] Søgaard H T, Olsen H J. Determination of crop rows by image analysis without segmentation. Computers and Electronics in Agriculture, 2003;
38(2): 141-158

[3] Kaizu Y, Yokoyama S, Imou K, Nakamura Y. Vision-based navigation of a rice transplanter. Collection of Extent Abstracts of 2004 CIGR International Conference, 2004.

[4] Marchant J A, Brivot R. Real-time tracking of plant rows using a Hough transform. Real-time Imaging, 1995; 1(5): 363-371.

[5] Marchant J A. Tracking of row structure in three crops using image analysis. Computers and Electronics in Agriculture, 1996; 15(2): 161-179.

[6] Yuan Z Y, Mao Z H, Wei Q. Crop rows positioning technology based on computer vision. Journal of China Agricultural University, 2005; 1(3): 69-72. (in Chinese)

[7] Zhang Q, Huang X G, Li B. Centerline detection based on color model and nearest neighbor method paddy seedlings columns clustering. Transactions of the CSAE, 2012; 28(17): 163-171. (in Chinese)

[8] Ding Y C, Wang S M, Chen H. Navigation path detection algorithm based on image rotation projection. Transaction of the CSAM. 2009; 40(8): 155-160. (in Chinese)

[9] Si Y S, Jiang G Q, Liu G, Gao R, Liu Z X. Early crop row center line detection method based on least squares method. Transaction of the CSAM, 2010; 41(7): 163-167. (in Chinese)

[10] Cao Q, Wang K, Li H. Dry multi-target line detection method based on machine vision. Transactions of the CSAE, 2010; (Supp): 187-191. (in Chinese)

[11] Chen B Q, Tojo S, Watanabe K, Ai F, Huang B K. Studies on the Computer-eye of Rice Transplant Robot. Journal of the Japanese Society of Agricultural Machinery, 1997; 59(3): 23-28. (in Janpanese)

[12] Chen B Q, Tojo S, Watanabe K. Machine vision based algorithmic guiding system for automatic rice transplanters. Applied Engineering in Agriculture, 2003; 19(1): 40-46.

[13] Chen B Q, Tojo S, Watanabe K. Detection Algorithm for traveling route in paddy field for automated managing machines. Transaction of the ASAE, 2002; 45(1): 239-246.

[14] Chen B Q, Tojo S, Watanabe K. Study on Machine Vision for Micro Weeding Robot in Paddy Field. Biosystems Engineering, 2003; 85(4): 393-404.

[15] Zhao Y, Chen B Q, Wang S M, Dai F Y. Fast detection of furrows based on machine vision on autonomous mobile robot. Transactions of the CSAM, 2006; 37(4): 81-86. (in Chinese)

[16] Zhang L, Wang S M, Chen B Q, Zhang H X. Crop-edge detection based on machine vision. New Zealand Journal of Agricultural Research, 2007; 50(5): 1367-1374.

[17] Zhang H, Chen B Q, Zhang L. Detection algorithm for crop multi-centerlines based on machine vision. Transactions of the ASABE, 2008; 51(3): 1089-1097.

[18] Li J B, Chen B Q, Liu Y. Study on image detection method of navigation route of the cotton plastic film mulch planter. Transactions of the CSAM, 2014; 45(1): 40-45. (in Chinese)

[19] Li J B, Chen B Q, Liu Y, Cha T. Detection for navigation route for cotton harvester based on machine vision. Transactions of the CSAE, 2013; 29(11): 11-19. (in Chinese)

[20] Haw C L, Ismail W I W, Kairunniza-Bejo S, Putih A, Shamshiri R. Colour vision to determine paddy maturity. Int J Agric \& Biol Eng, 2014; 7(5): 55-63. 